\title{
Modelo estructural de las relaciones entre enseñanza en contextos tecnológicos y el rendimiento académico en Contabilidad
}

\author{
Structural model of the relationships between teaching in tech-nological contexts \\ and academic performance in accounting
}

Recibido: 5 de marzo 2020 / Aprobado: 24 de mayo de 2020

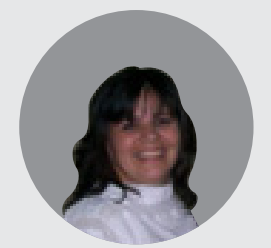

IDALIA GABRIELA DE CASTRO UNIVERSIDAD NACIONAL DEL NORDESTE idecastro@eco.unne.edu.ar

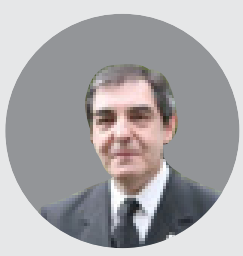

ANTONIO HUMBERTO CLOSAS UNIVERSIDAD NACIONAL DEL NORDESTE hclosas@hotmail.com

\section{Resumen}

La relevancia de las relaciones entre modalidad de enseñanza, uso de tecnologías y resultados educativos está dada por su impacto en el desarrollo humano, la importancia en la agenda pública, su vacancia científica y la trascendencia local. En este marco, el objetivo del presente trabajo radica en proponer un modelo, logrado mediante la técnica estadística estructuras de covarianza, que refleje de qué manera y en qué medida la enseñanza a través de materiales multimedia se vincula con el desempeño académico de estudiantes universitarios. El anclaje institucional ha posibilitado la extracción aleatoria de la muestra aceptante, conformada por 118 estudiantes (mujeres 60.17\%), con edad media de 21.08 años (DE = 3.76), que asistieron en el año 2017 a la Facultad de Ciencias Económicas de la Universidad Nacional del Nordeste, Argentina. El diseño metodológico es inicialmente explicativo, de naturaleza cuasiexperimental y descriptivo mediante encuesta, es predominantemente un estudio de línea cuantitativa. El desarrollo de la investigación permite proponer un modelo teórico que es contrastado a nivel empírico, dando lugar a una representación ajustada a los datos. En esta figura es posible observar la influencia y riqueza de la modalidad de enseñanza semipresencial en la formación disciplinar de los alumnos en una asignatura del área contable. El modelo final propuesto sería un recurso válido para evaluar la eficacia de la implementación de nuevas estrategias de enseñanza en el ámbito local de educación superior.

Palabras clave: Modalidad semipresencial; rendimiento; herramientas tecnológicas; modelo.

\footnotetext{
a. Magíster en Metodología de la Investigación Científica. Especialista en Contabilidad Superior y Auditoria. Profesora Adjunta, con dedicación exclusiva, en la cátedra Fundamentos de Contabilidad de la Facultad de Ciencias Económicas de la Universidad Nacional del Nordeste. Investigadora categoría III del Programa de Incentivos del Ministerio de Educación de la Nación Argentina. Coautora de libros y de publicaciones en revistas científicas nacionales y extranjeras. Para contactar a la autora: idecastro@eco.unne.edu.ar

b. Doctor en Estadística e Investigación Operativa por la Universidad Pública de Navarra (Pamplona, España). Profesor Titular de Estadística II y Director de Proyectos de Investigación en la Facultad de Ciencias Económicas de la Universidad Nacional del Nordeste. Investigador categorizado del Programa de Incentivos del Ministerio de Educación de la Nación Argentina. Coautor de libros y de diversas publicaciones en revistas de impacto científico nacionales y extranjeras. Para contactar al autor: hclosas@ hotmail.com
} 


\begin{abstract}
The relevance of the relationships between teaching modality, use of technology and academic performance, is given by its impact on the human development, its importance on the public agenda and, at the same time, the scientific vacancy and the local transcendence of this topic. Accordingly, the aim of this research is to propose a model, achieved through the statistical technique of covariance structures, that reflects how and to what extent teaching through multimedia materials is linked to the academic performance of university students. The institutional anchoring has enabled the random extraction of the accepting sample which consisted of 118 students (60.17\% women), $M=21.08$ years, $(S D=3.76)$, who attended classes at the School of Economic Sciences of the National Northeastern University, Argentina, in 2017. The methodological design is mainly explicative, quasi-experimental and descriptive by means of survey, predominantly a quantitative line study. The development of the research allows to propose a theoretical model that is contrasted at the empirical level, giving rise to a representation adjusted to the data. In this figure it is possible to observe the influence and richness of the blended learning modality in the disciplinary formation of the students, in a subject of the accounting area. The proposed final model would be a valid resource to evaluate the effectiveness of the implementation of new teaching strategies in the local area of higher education.
\end{abstract}

Keywords: blended modality; performance; technological tools; model.

\title{
1. Introducción
}

Es indudable que independizar la enseñanza del aprendizaje constituye un verdadero desacierto didáctico; de igual manera, desligar totalmente la labor docente del resultado académico obtenido por el alumno sería una consideración absolutamente errónea (Zabalza, 2002). Si bien el estudiante es responsable de su desarrollo, se hace necesario repensar la enseñanza para mejorar los resultados de aprendizaje, mucho más si se tiene presente que los estudiantes de nuestras aulas comparten las características de los nativos digitales (Gallardo, Marqués y Bullen, 2014; García, Portillo, Romo y Benito, 2007).

El uso de la tecnología permite responder necesidades de formación de los jóvenes actuales y diversificar el formato de la oferta educativa universitaria en sus distintas modalidades: presencial, no presencial (virtual o e-learning) o mixta (semipresencial o b-learning). En razón de lo que antecede, y dado que Contabilidad Básica es una asignatura de primer año, en el contexto académico que tienen lugar esta investigación, se seleccionó la modalidad semipresencial (MSP) como oferta educativa adecuada al grupo de alumnos a la que está destinada, a efectos de su análisis.

El abordaje del tema objeto de estudio (relaciones causales entre modalidad de enseñanza semipresencial, mediada por TIC, y la formación disciplinar de los alumnos, en una asignatura del área contable) es consistente con el paradigma constructivista y las teorías del aprendizaje centradas en el alumno, con referentes indiscutibles como: Piaget, Vigotsky y Ausubel. La importancia de recuperar sus bases deviene de su relación con las teorías educativas y de la función que concede a factores sociales en la explicación de un hecho. En un segundo plano resultan pertinentes aquellos aspectos técnicos específicos sobre tecnología y contabilidad. 


\subsection{Planteamiento del problema}

En los distintos niveles educativos es una realidad comprobada la notable diferencia que existe entre el rendimiento académico deseado y el obtenido por los estudiantes. Sin embargo, resulta evidente que cualquier solución que se proponga para este fenómeno multicausal será tan compleja como su génesis, y su éxito no dependerá exclusivamente del docente y/o del alumno e interpelará al proceso educativo para introducir cambios que, hasta ahora, han resultado insuficientes para resolver eficazmente este problema (De Castro, Closas y Cruz, 2019).

La situación cotidiana del rendimiento en las aulas universitarias es altamente preocupante, debido, entre otras razones, a la notable distancia que existe entre los conocimientos reales que poseen los estudiantes y los que resultan necesarios para lograr que el proceso de enseñanza-aprendizaje se desarrolle de manera óptima y consiga la acreditación esperada.

La realidad descrita, en algunas ocasiones, trasciende a las asignaturas del ciclo de especialización y tiene consecuencias no deseadas en el desempeño académico y la formación profesional de los educandos, dado que las carreras organizan su plan de estudios y las materias su contenido, como es lógico, bajo el supuesto de gradualidad y correlatividad. Este hecho ha sido observado y constatado por los autores de este trabajo en virtud de ejercer la actividad docente en asignaturas del ciclo básico común, que se imparten en el ámbito donde se realiza el presente estudio.

Otro problema que presenta hoy en día la educación es el relacionado con la simultaneidad en la ocurrencia de la enseñanza y el aprendizaje. Es indiscutible que existe un desarrollo de la vida en tiempo real, pero a la vez, es innegable que los estudiantes se desenvuelven y disfrutan de aprender a través de entornos virtuales de manera asincrónica.
Esta realidad se convierte en un problema para una oferta formativa de modalidad presencial y, específicamente, cuando los docentes utilizan métodos tradicionales de enseñanza, dada la distancia entre la amplitud e intensidad de uso de la tecnología que tienen los alumnos en relación con el escaso uso que, por lo general, hacen de ella sus profesores.

Zempoalteca, Barragán, González y Guzmán (2017) presentan en su estudio varias causas por las cuales los docentes se resisten a la innovación tecnológica: por la costumbre a sus metodologías tradicionales, pues nuevos métodos requieren mayor esfuerzo; porque la evaluación en las instituciones de educación superior sigue respondiendo a sistemas tradicionales, y lo mismo sucede con muchos de los programas o currículos vigentes.

La importancia, prioridad y esfuerzo que las instituciones de educación superior depositen en investigar las relaciones entre enseñanza, aprendizaje, uso de tecnologías y rendimiento académico partirán del impacto que estas asociaciones presentan en el nivel de progreso que tienen los países (medido a través del índice de desarrollo humano, IDH); la relevancia otorgada en la agenda pública nacional e internacional (evaluaciones estandarizadas de aprendizaje) y la invisibilidad del tema (carencia de estudios en el área pedagógica).

Además de esos objetivos macros, este estudio se justifica por la vacancia científica (el fenómeno del rendimiento en la disciplina contable es una temática poco explorada por la investigación académica), y el interés propio de aportar datos que arrojen luz a la problemática del bajo rendimiento académico por la escasa satisfacción que provocan las metodologías tradicionales (de acuerdo con De Castro et al., 2019, diversas experiencias previas han evidenciado la atracción de los estudiantes por ofertas educativas con mayor incidencia tecnológica).

Este estudio está dirigido a evaluar la 
eficacia global del proceso educativo cuando este se caracteriza por el uso de tecnologías de la información y la comunicación (TIC). Para ello se plantea un modelo, a partir de algunos aspectos del ámbito psicoeducativo, que expresa las relaciones entre las variables involucradas en la investigación, que es contrastado empíricamente por medio del método explicativo del análisis multivariado, denominado ecuaciones estructurales (conformado por las técnicas análisis factorial confirmatorio y modelos de estructuras de covarianza).

En líneas generales, el método estadístico se destaca por su carácter confirmatorio respecto del modelo que se postula, que surge del planteo de una hipótesis teóricamente pertinente en el contexto de interés, presentando la importante cualidad de tener la capacidad de generar constructos que estiman las variables latentes que se reflejan en las variables medibles, para posteriormente calcular los parámetros especificados por las relaciones propuestas a nivel hipotético. Otro hecho para señalar es que a las variables dependientes, sean estas observadas 0 latentes, se las mide teniendo en cuenta su error residual.

Las preguntas que nos hacemos y que servirán de guía para este estudio son: ¿Cómo impacta la implementación de la modalidad semipresencial, así como el uso de herramientas y actividades de la Web 2.0 en el rendimiento de los estudiantes de la asignatura de Contabilidad Básica? El hecho de ofrecer nuevas estrategias más acordes a las necesidades y naturaleza de los estudiantes, ¿contribuye a su satisfacción, motivación y, por ende, a su mejoría en el rendimiento académico? ¿Qué modelo estadístico es idóneo para identificar en qué manera y en qué medida la enseñanza a través de materiales multimedia se vincula con el desempeño académico de estudiantes universitarios?

Así pues, el enunciado formal del objetivo principal de este estudio expresa: proponer un modelo estadístico, elaborado a través de estructuras de covarianza, que muestre la relación entre los factores predictivos enseñanza, materiales y aprendizaje, mediados por TIC, y el rendimiento académico de estudiantes universitarios, en el ámbito de la asignatura Contabilidad Básica (CB).

Este trabajo se encuentra dividido en varias secciones. La primera parte responde a la fase introductoria en la que se realizó una descripción del problema de investigación; a continuación, presentamos breves rasgos de la modalidad semipresencial, también los factores y variables a considerar; la segunda parte se relaciona con el estudio empírico, en cuyo marco se han proporcionado los resultados. Finalmente, ofrecemos la discusión y las conclusiones que detallan las fortalezas y debilidades del modelo estadístico utilizado para medir el impacto en el rendimiento de la modalidad semipresencial en cuanto a las características de su relación con la enseñanza, los materiales y el aprendizaje, y la reflexión que de ello se desprende.

\subsection{La modalidad semipresencial o el b-learning}

Troncoso, Cuicas y Debel (2010) realizaron un estudio con el propósito de presentar el resultado de la implementación del modelo b-learning en la enseñanza de la asignatura Matemática I en la carrera de Ingeniería Civil de la UCLA, Venezuela. Los autores definen el b-learning como "un modelo educativo que ofrece de manera sistémica una combinación o mezcla óptima de recursos, tecnologías y medios tecnológicos de aprendizaje virtual y no-virtual, presencial y a distancia, en diversas proporciones, combinaciones y situaciones, adecuándolas a las necesidades educativas" (p. 5).

La investigación mencionada presenta las ventajas del b-learning y hace énfasis en que pudiera considerarse una modalidad superior a la presencial y a la completa- 
mente en línea (e-learning) por la equilibrada combinación que hace de ambas. A saber, le saca el mayor provecho a las herramientas y actividades de la Web 2.0 tan valiosas para desarrollar competencias colaborativas; también ofrece ventajas por la acción tutorial que ejerce el docente, lo cual añade cercanía y un aprendizaje en un ambiente más apropiado; además, su gran mérito consiste en que supera la barrera de tiempo y espacio. Sin embargo, esta modalidad semipresencial también aprovecha el beneficio de la presencialidad, pues no desecha el valioso rol del docente instructor de los encuentros presenciales donde los estudiantes fomentan la capacidad de escucha atenta, pueden exponer ante una audiencia auténtica, experimentar en situaciones reales y debatir ante grandes grupos, en definitiva, donde la zona de desarrollo se vuelve más próxima por la interacción humana.

Otras características de la modalidad semipresencial, según Bartolomé (2008), Cabero y Llorente (2008), citados por Troncoso et al. (2010), se refieren al papel activo de su aprendizaje que asume el estudiante y al rol del docente como mediador y dinamizador. Además de que ofrece un abanico de "tipologías de comunicación que propician la interacción de manera sincrónica, asincrónica, tutoría presencial, comunicación textual, auditiva, visual y audiovisual". Este último rasgo es esencialmente particular para los jóvenes, los denominados la generación de los milenios, quienes han crecido con una necesidad impuesta de estar siempre conectados, valoran la cercanía a través de las redes y tienen un fuerte apego a todo lo tecnológico.

Debido a este apego de los jóvenes a la tecnología y el uso masivo que hacen de ella, muchas investigaciones recientes se enfocan en medir su impacto en el aprendizaje. García-Martín y Cantón-Mayo (2019) refieren un estado del arte en este sentido y presentan las herramientas que ofrece la Web 2.0 como "nuevos determinantes del rendimiento académico ya que inciden en el trabajo del estudiante a distintos niveles y de diferentes formas". Las autoras detallan la multiplicidad de estudios y en qué se enfoca cada uno. Entre ellos, nos llama la atención el de Cetinkaya (2017), así como Noshahr, Talebi y Mojallal (2014), cuyas investigaciones muestran que la comunicación directa e individualizada, provocada por la mensajería instantánea, incrementa la confianza y la sensación de intimidad entre los estudiantes y sus docentes, lo que provoca a su vez un aumento en el rendimiento académico.

En esta misma línea del rendimiento, Gil (2012) y Torres-Díaz et al. (2016), citados por García-Martín y Cantón-Mayo (2019), han llegado a la conclusión de que en la educación superior los jóvenes que hacen un uso equilibrado de las tecnologías para búsquedas en fuentes adecuadas, no solo mejoran su rendimiento académico, sino que alcanzan mayor éxito y desempeño. Es evidente que la potencialidad de las TIC para el aprendizaje está estrechamente relacionada con las posibilidades que ofrecen para representar, procesar, transmitir y compartir datos. Sin embargo, la información se convierte en conocimiento y su acceso da lugar al aprendizaje cuando actuamos sobre ella, la procesamos, la organizamos, nos la apropiamos, la utilizamos y la confrontamos; en suma, cuando le damos significado y sentido (Coll, 2004, citado por De Castro et al., 2019).

A continuación, presentamos una breve descripción de los factores y las variables que tomamos en cuenta para elaborar el modelo estadístico logrado mediante la técnica estructuras de covarianza, que mostrará de qué manera y en qué medida la enseñanza a través de materiales multimedia se vincula con el desempeño académico de estudiantes universitarios.

\subsection{Algunos aspectos sobre los factores y las variables que intervienen en el modelo}

El triángulo didáctico (integrado por los elementos enseñanza, aprendizaje y 
contenidos), siguiendo a Coll (2004), citado por De Castro et al. (2019), ha sido un potente marco doctrinario orientador para yuxtaponer los consensos teóricos y la realidad fáctica de la MSP representada en los datos de la muestra. A su vez, el principio de causalidad (en este caso conformado por, enseñanza, materiales y aprendizaje -causas- y rendimiento académico -efecto-), brindó una manera asequible de organizar el terreno empírico, a través de una serie de pautas razonables que resultaban conocidas desde nuestra praxis docente.

La enseñanza ha sido una acción intencionada, planificada, organizada, dirigida, sistemática $\mathrm{y}$, en este caso, mediada por TIC, para compartir conocimientos; y la estrategia adoptada para promover en el alumno el acceso intelectual a los mismos como su apropiación significativa ha sido la MSP.

La oferta educativa ha requerido la realización de actividades que implican la presencia simultánea en un aula de estudiantes y profesores en valores de tiempo cercanos al cincuenta por ciento (50\%). El resto del tiempo se ha destinado a actividades de enseñanza reguladas, gestionadas a distancia y con autorregulación del aprendizaje. La incorporación del componente tecnológico al proceso ha requerido de los docentes-tutores nuevas habilidades, no siempre comparables con las utilizadas en los sistemas presenciales.

Los contenidos han ingresado al modelo didáctico-operativo a través de los tipos de materiales empleados (p. ej., cuestionarios), predominantemente multimedia, donde actividades y recursos compartidos en plataforma Moodle, presentaciones Prezi, trabajo colaborativo en Wiki, almacenamiento de archivos en Google Drive, videos de Youtube y aplicación educativa gratuita Kahoot!, son algunas de las herramientas de la Web 2.0 que se han incorporado al dictado de CB en la MSP para impulsar el proceso cognitivo en el alumno con el objeto de generar un aprendizaje significativo. La eficacia del modelo mencionado se halla asociada con la selección pertinente en correspondencia con los contenidos temáticos del programa, la aptitud pedagógica para generar interacción en el aula virtual, la potencialidad técnica en la formación profesional y la receptividad metodológica por parte de los estudiantes.

El aprendizaje observado a través de la interacción con los materiales y la satisfacción del alumno con el proceso educativo, como también el rendimiento académico, forman parte de sus resultados.

En cuanto al rendimiento académico, se ha adoptado la decisión operativa generalizada de medirlo a través de las calificaciones, tanto por las ventajas que se derivan del uso de indicadores estandarizados (p. ej., comparabilidad, estabilidad y fiabilidad), como por decisiones propias del equipo de investigación, aunque se han reconocido sus limitaciones objetivas y subjetivas.

En particular, se ha asumido que la media aritmética teórica calculada a partir de las calificaciones obtenidas en exámenes escritos previstos en el régimen de Pruebas Parciales Acumulativas (PPA), resume la complejidad del proceso educativo dando una visión global, completa y contextualizada del desempeño del estudiante. Sin embargo, la calificación final real asignada al alumno resulta de considerar, además del promedio, el cumplimiento de condiciones reglamentarias. En virtud de ello, la media aritmética teórica y la calificación real pueden diferir.

Se considera que la media aritmética teórica podría ser un ratio representativo del aprendizaje producido por la interacción con los contenidos; en tanto, la calificación real sería un indicador más adecuado para ponderar el rendimiento académico y un sensor más estricto en la evaluación global de la MSP. 


\section{Materiales y Método}

\subsection{Participantes}

Este estudio se realizó en la Facultad de Ciencias Económicas de la Universidad Nacional del Nordeste, Argentina, específicamente en la asignatura de Contabilidad Básica, la cual es común a las carreras de: Contador Público (CP), Licenciatura en Administración (LA), Licenciatura en Economía (LE) y Licenciatura en Comercio Exterior, que se imparte en cuatro sedes, emplazadas en las ciudades de Resistencia, General Pinedo, Goya y Paso de los Libres (De Castro et al., 2019).

En primer lugar, se ha definido el criterio (sede Resistencia, régimen de PPA y enseñanza en la MSP) para seleccionar los sujetos que podrían facilitar información. Posteriormente la aplicación de un cuestionario ad hoc -diseñado para recolectar datos sobre el nivel de satisfacción de los estudiantes que participaron de la MSP, respecto del proceso enseñanza-aprendizaje- definió la conformación aleatoria del grupo definitivo de sujetos.

Así, la muestra aceptante (Fox, 1981) quedó conformada por jóvenes de ambos sexos, con edades comprendidas entre 18 y 38 años, que respondieron de manera volun- taria al cuestionario mencionado, los cuales proporcionaron la información para las diversas pruebas estadísticas que se realizaron. En concreto, se analizaron los datos relativos a un total de 118 estudiantes $(71$ mujeres, $60.17 \%$ y 47 hombres, $39.83 \%$ ), con una media de 21.08 años y desviación estándar de 3.76, que asistieron en el curso académico 2017.

\subsection{Diseño}

El diseño metodológico de esta investigación es inicialmente explicativo, de perfil correlacional y mediacional, de naturaleza cuasiexperimental, aunque por momentos solo observacional; puesto que, sin manipular las variables, excepto el llamado control estadístico, se reparó en el fenómeno objeto de interés tal y como se da en su contexto real para después analizarlo.

Es también un estudio de estilo descriptivo mediante encuesta, se empleó la técnica del cuestionario en el marco de la investigación de campo, la cual responde a una estrategia de tipo longitudinal. Además, es un trabajo de línea prospectiva, dado que se buscó evaluar relaciones entre las variables que intervienen, para proyectar recomendaciones que resulten viables y sustentables en el tiempo.

Por último, debemos señalar que el estudio posee un enfoque multimodal, donde prevaleció el aspecto cuantitativo y su diseño es fácticamente posible y científicamente admitido en trabajos de estirpe constructivista afiliados a la línea de investigación consolidada dentro del cognitivismo, sobre aprendizaje significativo (Nicolás, 2018; Chávez, 2018).

\subsection{Procedimiento}

La recolección del conjunto de datos provino de instrumentos de observación de diferentes características, y por ello el procedimiento de aplicación ha sido adecuado a su mejor desarrollo posible en cada caso.

En líneas generales, la técnica de recolección empleada ha sido la encuesta administrada en forma personal, a través de plataforma (Moodle y SIU Guaraní) o por correo electrónico, la cual contenían ítems sobre distintos aspectos del grupo-clase y su contexto, que fueron aplicados a estudiantes y docentes; los detalles pueden observarse en la Tabla 1.

La organización incluyó el análisis inicial de las variables, la definición de la escala de medición compatible con su naturaleza; en tanto durante el procesamiento se tabularon las observaciones recogidas del trabajo de campo en un archivo de Microsoft Excel 
donde se las ordenó, vinculándolas con cada alumno de la muestra, generando así una matriz electrónica de datos. Los datos personales de los estudiantes (unidades de análisis) fueron indicados en filas, en tanto que las variables de cada sujeto (p. ej., sexo, calificación) se anotaron en columnas.

\subsection{Instrumentos, factores y variables}

La captura de datos muestrales mediante cinco (5) instrumentos estadísticos de medición distintos (ver Tabla 1) ha sido, por cierto, una tarea compleja. Aunque cabe señalar que previamente a su aplicación, y en los casos que correspondía (p. ej., Cuestionario sobre modalidad semipresencial), fue examinada su validez cualitativa (capacidad de medir lo que realmente se observa), mediante el juicio de expertos con experiencia en educación universitaria y/o enseñanza contable. Se puede afirmar que las apreciaciones respecto del contenido de los ítems propuestos y de la conformación factorial o estructural de los instrumentos evaluados, tuvieron una coincidencia promedio, en valores porcentuales, muy aceptable. Los análisis realizados en la línea de validez cualitativa resultaron favorables a efectos de minimizar los márgenes de error al momento de la utilización de las pruebas en el espacio educativo propuesto para esta investigación, durante el trabajo campo.

Ahora bien, una vez que fue posible aplicar los instrumentos, recoger la información y construir la base de datos en formato electrónico, fue ponderada su confiabilidad (cuantía en que las medidas de las pruebas están libres de errores casuales 0 aleatorios), a través de indicadores del ámbito de la psicometría (coeficientes de correlación dimensión-total corregida y de consistencia interna). El procesamiento de los datos fue realizado, en esta ocasión, con ayuda del programa IBM SPSS Statistics 22.
En concreto, y muy brevemente, los documentos de observación empleados fueron:

(1) Escalas para la evaluación interactiva del proceso de enseñanzaaprendizaje: este material, elaborado por De la Fuente y Martínez (2004), tiene el propósito de posibilitarle al docente reflexionar sobre el nivel de satisfacción respecto de su desempeño educativo en el grupoclase.

(2) Informe de reseña docente: fue especialmente diseñado para que el educador recapacite sobre el conjunto de decisiones operativas que conlleva el proceso de E-A, durante el dictado de la asignatura.

(3) Reportes de plataforma: permiten el seguimiento individualizado, sistemático, continuo e integral del estudiante dentro del aula virtual.

(4) Cuestionario sobre modalidad semipresencial: este instrumento ad hoc fue diseñado para recolectar datos sobre el nivel de satisfacción de los estudiantes que participaron de la MSP, respecto del proceso de E-A.

Actas de calificaciones de exámenes: documentos oficiales donde consta la calificación obtenida por cada estudiante en los exámenes parciales escritos de la asignatura y su situación académica al finalizar el dictado del semestre.

En tato que, algunos aspectos de las doce (12) variables que han sido relevadas a través de los instrumentos que se acaban de enunciar, se describen a continuación. Se comienza por detallar los indicadores relativos al factor o constructo enseñanza.

- Satisfacción con el proceso de enseñanza (SPE): es una dimensión, conformada por siete (7) ítems, que 
forma parte del instrumento Escalas para la evaluación interactiva del proceso de enseñanza-aprendizaje (EIPEA).

- Satisfacción con el proceso de aprendizaje (SPA): ídem al anterior, solo que esta subescala se encuentra conformada por catorce (14) ítems.

- Reseña docente aspectos positivos (RDAP): a través de este instrumento se documenta la opinión de docentes de la materia sobre distintas cuestiones del proceso de enseñanza. Su estructura es simple y, en este estudio, responde a la solicitud: Por favor, comente brevemente los aspectos positivos que, a su criterio, resultaron importantes en la implementación del dictado de la asignatura Contabilidad Básica.

Las siguientes variables forman parte del instrumento Reportes de plataforma, están disponibles para consulta en línea o exportación en archivos del programa Excel. Se han considerado datos del informe del calificador que registró la valoración numérica de las actividades y el informe de progreso que asentó el estado cualitativo (finalizado-fecha/no finalizado) de actividades y recursos empleados. En particular, se ha trabajado con cuatro (4) conjuntos de datos para el constructo o variable latente materiales:

- Material de lectura (ML): texto, elementos multimedia o enlaces que presentan la información de cada unidad del programa.

- Cuestionarios (CUE): preguntas con distinto formato de respuesta (opción múltiple, verdadero/falso, coincidencia, etc.).

- Tareas (TAR): presentaciones de los alumnos que el docente revisa, valora y califica pudiendo brindar retroalimentación y nuevos plazos de presentación.

- Foros (FOR): discusiones asincrónicas durante un período de tiempo prolongado mantenidas por los participantes (alum- nos y docentes).

Las variables observadas que continúan fueron las que conformaron el diseño del constructo aprendizaje.

- Cuestionario sobre modalidad semipresencial (CMSP): Estuvo compuesto de cuatro (4) preguntas cerradas y dos (2) abiertas, que capturaron datos sobre la situación socioeconómica, motivación, materiales educativos y satisfacción de

Tabla 1. Operacionalización de variables observadas y latentes

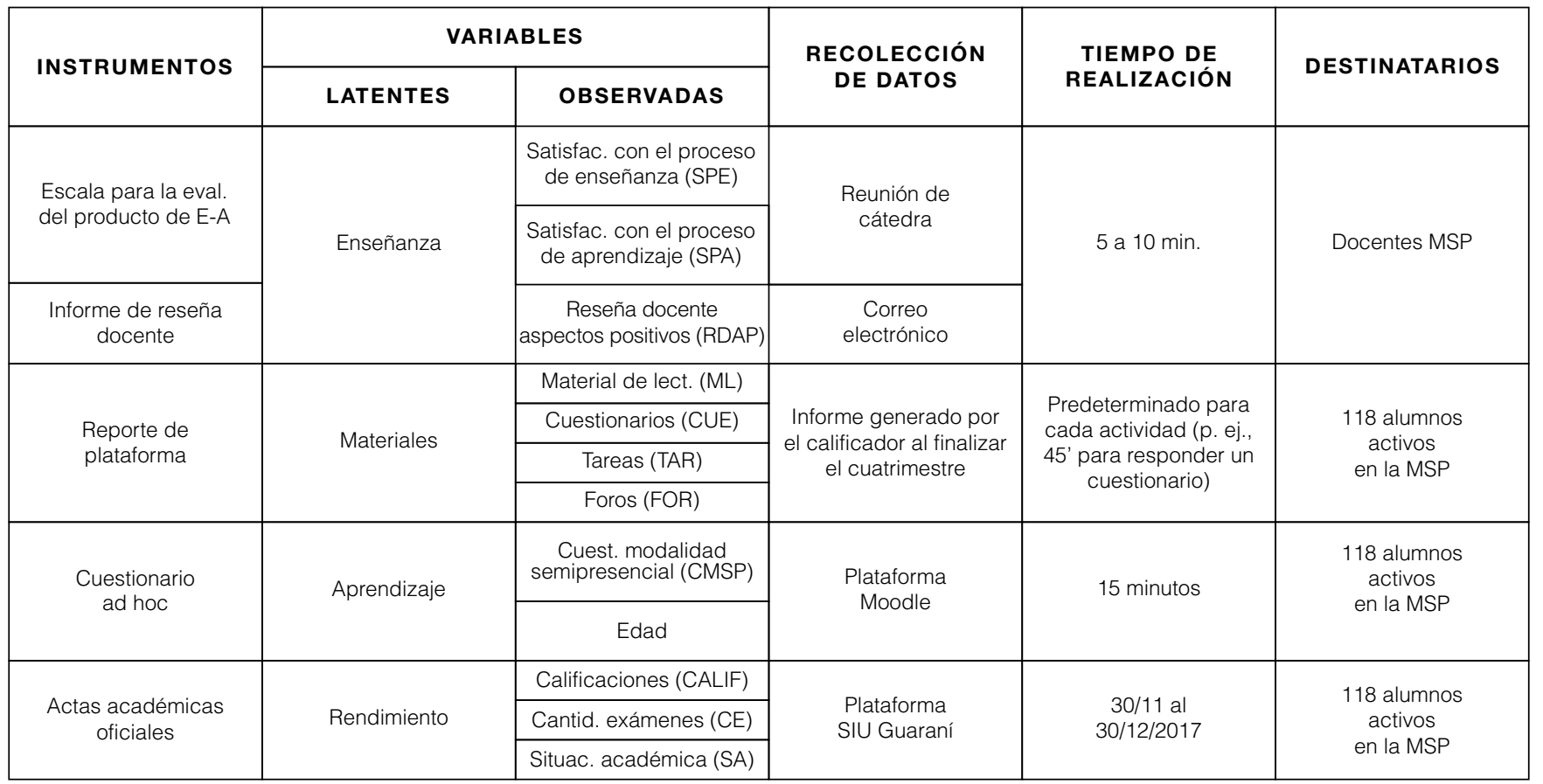


los educandos con el dictado de la asignatura CB.

- Edad: esta característica etaria fue recogida del CMSP, en razón que la aplicación del mismo fue nominada, además de voluntaria y aleatoria.

Finalmente se presentan las variables, vinculadas con el factor rendimiento, cuyos datos fueron obtenidos a través de las actas académicas que se encuentran disponibles en la plataforma oficial SIU (Sistema de Información Universitaria) Guaraní.

- Calificaciones (CALIF): valor medio correspondiente a las notas obtenidas en instancias (tres en promedio) de pruebas escritas parciales acumulativas teórico-prácticas.

- Cantidad de exámenes (CE): número total de pruebas parciales escritas realizadas por el estudiante durante el cursado de la asignatura.

- Situación académica (SA): atributo conceptual representativo del resultado global del alumno, asignado al finalizar el semestre lectivo.

\subsection{Análisis de datos}

En virtud del objetivo que se pretende lograr en este estudio, y a efectos de examinar si las relaciones que conforman el modelo teórico (véase Figura 1) se ajustan a los datos empíricos de la investigación, se utilizó el análisis de ecuaciones estructurales del programa EQS 6.3 (Bentler, 2006; Bentler y Wu, 2015). En el procedimiento de estimación se trabajó con el método de máxima verosimilitud ( $M L$, Maximum Likelihood), dado que se consideró razonable asumir la existencia de normalidad en la distribución de las variables observadas, pues la estimación normalizada del coeficiente de Mardia -indicador de la curtosis multivariante alcanzó un valor de 2.930, inferior al criterio de máxima (normalized estimate $=5$ ) recomendado (Bentler, 2006).
La evaluación del modelo se realizó a través de: a) estudio analítico, a efectos de determinar y contrastar las relaciones entre las variables postuladas en las hipótesis; y b) análisis de su grado de ajuste global, con el fin de comprobar en qué medida el modelo teórico reproduce correctamente las relaciones existentes en la matriz de correlaciones de datos empíricos.

En efecto, la formulación algebraica correspondiente con el fin de estimar los coeficientes, parámetros y evaluar estructuralmente el modelo propuesto ha sido planteada a partir del gráfico teórico representado en la Figura 1. En ella, los términos de error de las variables observadas tienen asignada la etiqueta $E$, mientras que los errores de las estimaciones de las variables latentes (constructos), no independientes, se indican con la letra $D$ (de disturbance).

Posteriormente a la valoración inicial del modelo teórico mediante el método de $M L$, se han estimado los errores típicos y se procedió a la determinación del índice de ajuste utilizando el test de $\mathrm{X}^{2}$ (Satorra $\mathrm{y}$ Bentler, 1988), todo lo cual se detalla en la siguiente sección.

En las ecuaciones que se emplearon en el estudio analítico fueron fijadas arbitrariamente en 1 determinadas cargas factoriales entre variables observadas y latentes (RDAP y Enseñanza, TAR y Materiales, EDAD y Aprendizaje, CALIF y Rendimiento), al igual que los coeficientes de regresión, tanto de las variables observadas como de las variables latentes mediadoras (Materiales y Aprendizaje) y explicada (Rendimiento), respecto a los términos de error ( $E_{\text {RDAP, }} E_{S P E}, E_{S P A} ; E_{T A R}, E_{M L}, E_{C U E}, E_{F O R}$;

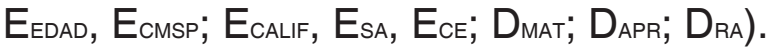

Por otra parte, se dejaron libres de valoración las varianzas de la valatente independiente (Enseñanza) y las varianzas de los errores de las variables observadas, así como las perturbaciones (disturbances) de los factores dependientes (mediadores, 
Materiales y Aprendizaje; y explicado, Rendimiento).

También se dejaron libres para ser estimadas las covarianzas entre los errores (E $E_{M L}$ y $E_{C U E} ; E_{S A}$ y $E_{C E}$ ) correspondientes a los respectivos indicadores de las variables latentes mediadora (Materiales) y explicada (Rendimiento); en cambio, se valoraron nulas la totalidad de aquellas covarianzas que en la Figura 1 no se encuentran indicadas.

\section{Resultados}

\subsection{Identificación del modelo}

Para estimar el modelo propuesto se dispone de $q \times(q+1) / 2=12 \times 13 / 2=78$ datos, que se corresponden con las varianzas-covarianzas de las doce variables observadas (q). A su vez, el número de parámetros que tendríamos que estimar es 51, diferenciados del siguiente modo: 12 varianzas de los errores $E$, 3 varianzas de los errores $D, 1$ varianza de factor independiente, 12 coeficientes de regresión de las variables observadas sobre los errores $E, 3$ coeficientes de regresión de los factores dependientes sobre los errores $D, 2$ covarianzas entre los errores $E, 12$ coeficientes de regresión entre los factores y las variables observadas y 6 entre factores dependientes e independientes.

Sin embargo, al formalizar las 19 restricciones indicadas -que en resumen son: los 15 coeficientes de regresión de los errores $E$ y $D$, así como las 4 cargas factoriales (RDAP y Enseñanza, TAR y Materiales, EDAD y Aprendizaje, CALIF y Rendimiento)-, los parámetros a estimar resultan $51-(15+4)=32$. Evidentemente, tras todos estos argumentos nuestro modelo se encuentra identificado y presenta $78-32=46$ grados de libertad, por tanto, puede someterse a contraste empírico.

\subsection{Estimación y evaluación del modelo}

El estudio analítico de las relaciones entre las variables postuladas en el modelo reveló que tanto las cargas factoriales como los parámetros estructurales estimados son coeficientes estadísticamente significativos. En efecto, los doce pesos factoriales (varían de 0.237 a 0.992), en el marco del modelo de medida (conjunto de relaciones entre las

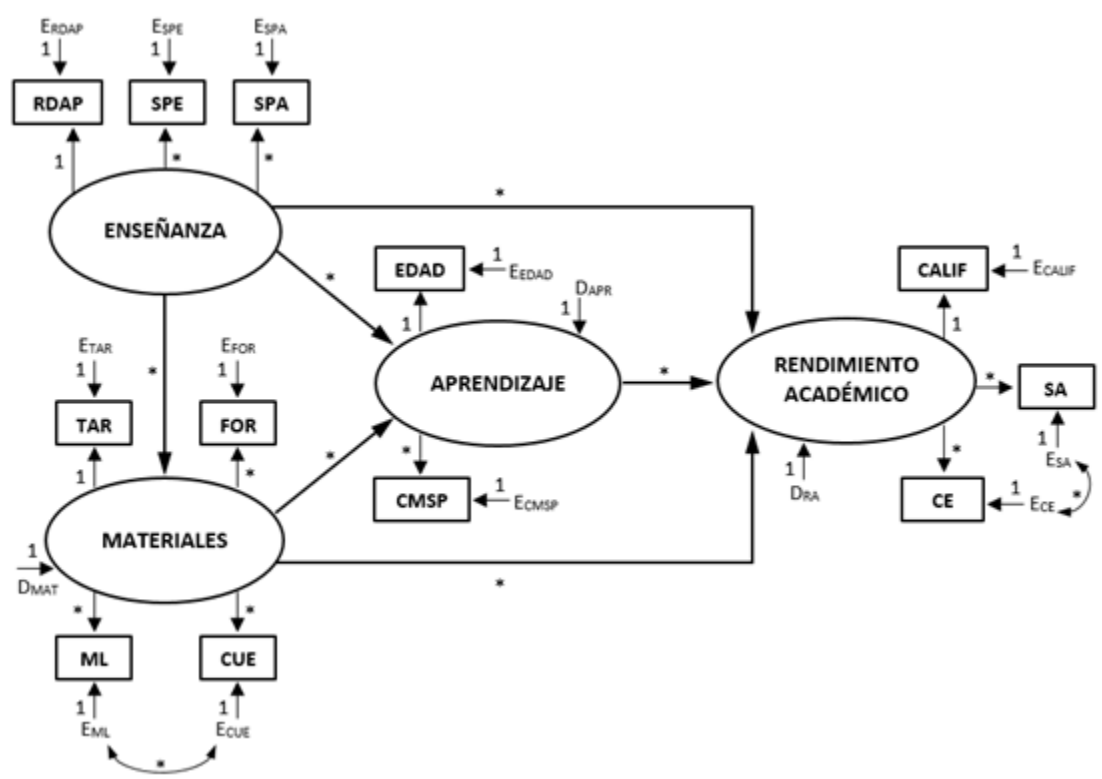

Figura 1. Modelo teórico de relaciones entre Enseñanza, Materiales, Aprendizaje y Rendimiento, elaborado mediante la notación de Bentler y Weeks (1980). 
variables observadas y la variable latente respectiva), resultaron estadísticamente significativos, algunos para $p<0.05$, y otros para $p<0.01$. Por lo tanto, pueden aceptarse las saturaciones obtenidas como indicios de validez de constructo de las diferentes variables latentes consideradas.

Asimismo, en el contexto del modelo estructural (conjunto de relaciones entre las variables latentes), los seis coeficientes de regresión entre el factor independiente y los factores dependientes (mediadores y explicado) que fueron estimados (Enseñanza y Materiales, Enseñanza y Aprendizaje, Enseñanza y Rendimiento; Materiales y Aprendizaje, Materiales y Rendimiento; Aprendizaje y Rendimiento), resultaron estadísticamente significativos (oscilan entre 0.237 y 0.616 ), algunos para $p$ $<0.05$, y otros para $p<0.01$.

También, los coeficientes que evalúan la covarianza entre los errores $E_{M L}$ y $E_{C U E}$, así como entre $E_{S A}$ y $E_{C E}$, resultaron estadísticamente significativos ( $a=0.05$ ). Creemos conveniente recordar que, en términos generales, una covarianza significativa indicaría que los factores asociados comparten una variación común que no ha podido ser explicada por las relaciones expresadas en el modelo.

Los diferentes valores originados como producto de las estimaciones realizadas en el marco del estudio analítico pueden verse en la Figura 2.

A efectos de juzgar el ajuste global del modelo, se ha tenido en cuenta, en primer lugar, la matriz residual de covarianzas (diferencia entre la matriz de covarianzas muestral y la matriz de covarianzas poblacional estimada), la cual en caso de que los valores de cada uno de sus elementos sean pequeños; esto es, cercana a una matriz nula, indicaría que el modelo ha sido capaz de ajustarse a los datos. Ahora bien, al examinar los residuos, es común observar el error promedio de los elementos estandarizados que se encuentran fuera de la diagonal; el cálculo de dicho valor en esta oportunidad ha resultado realmente bajo (0.052), indicando con ello un correcto ajuste.

En segundo lugar, siguiendo con el criterio de los residuos, fue posible comprobar que el $85.90 \%$ de estos caen dentro del intervalo $[-0.1,0.1]$, aunque no de forma simétrica (entre -0.1 y 0.0 se halla el $30.77 \%$, mientras que entre 0.0 y 0.1 está el $55.13 \%$,

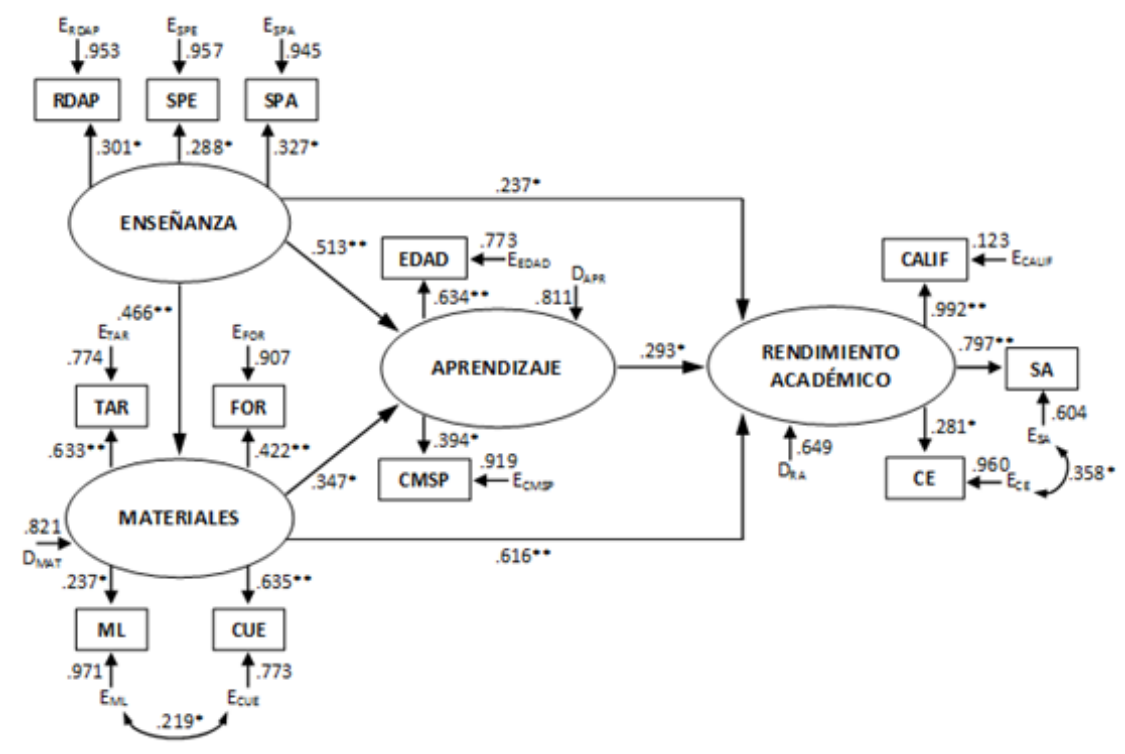

Nota. Grado de significación: ${ }^{*} p<0.05,{ }^{* *} p<$ 0.01

Figura 2. Resultados estandarizados del modelo propuesto para la intervención educativa en Contabilidad y en ambientes tecnológicos. 
de los valores residuales). En síntesis, se puede decir, a partir del análisis de los residuos, que el modelo teórico ha logrado bondad de ajuste.

Otro criterio que se valora mencionar, antes de exponer aquellos índices clásicos para juzgar globalmente el grado de ajuste, es el de la convergencia en el proceso de estimación. En efecto, dado que la estimación de un modelo es un proceso iterativo, el hecho de que el algoritmo converja de una manera rápida, es indicador de un buen ajuste. En nuestro caso, han sido necesarias diecinueve (19) iteraciones para la convergencia; sin embargo, el valor de la función de estimación (0.475) fue exactamente el mismo a partir de la número doce (12).

Para la evaluación global del modelo, de acuerdo con Schermelleh-Engel, Moosbrugger y Müller (2003), se ha utilizado una estrategia basada en los siguientes indicadores: el estadístico $\mathrm{X}^{2}$, junto con la razón entre este y los grados de libertad $\left(\mathrm{X}^{2} / g l\right)$, así como los índices descriptivos Comparative Fit Index (CFI), Non-Normed Fit Index (NNFI) y Root Mean Square Error of Approximation (RMSEA); todos los cuales no dependen tanto del tamaño muestral.

El test chi-cuadrado ha resultado, para un nivel $a=0.05$, estadísticamente no significativo, $X^{2}(46)=55.660, p=0.156$, y el cociente $X^{2} / g l=1.210$ razonablemente próximo a 1. A su vez, los índices $C F I$ y NNFI adoptaron valores 0.978 y 0.960 , respectivamente; mientras que la estimación puntual para la RMSEA fue 0.042, indicativos todos ellos de un buen ajuste entre el modelo y los datos.
A los índices de comparación estimados en primer término, se añaden otros estadísticos prácticos que proporciona EQS, entre los que se encuentran: Normed Fit Index (NFI) $=0.903$, Incremental Fit Index $(I F I)=0.982$ y McDonald's Fit Index $(M F I)=0.980$, los cuales también dejan en evidencia que el modelo asumido alcanzó bondad de ajuste, dado que igualan o superan el criterio de mínima (0.90) recomendado (Bentler, 2006). Una síntesis de los distintos estadísticos e índices relativos a la bondad de ajuste del modelo, originados a partir del método de $M L$, se encuentran detallados en la Tabla 2.

En resumen, a través de los distintos criterios e indicadores utilizados (análisis de los residuos, resumen de iteraciones $y$ contraste global), ha sido posible comprobar que la matriz de covarianzas observada y la predicha por el modelo propuesto no son significativamente diferentes; es decir, el modelo hipotetizado se ajusta al modelo empírico y, en consecuencia, sería de utilidad para explicar los datos.

Evidentemente, a partir de los resultados logrados, la adopción del modelo contrastado como modelo explicativo del fenómeno objeto de estudio es un hecho inmediato. En definitiva, ha sido posible alcanzar el objetivo planteado; esto es, proponer la modelización estadística del rendimiento académico en Contabilidad en contextos de enseñanza complementados por recursos multimediales, a través de la técnica estructuras de covarianza.

Tabla 2. Indicadores de bondad de ajuste global del modelo propuesto

\begin{tabular}{|c|c|c|c|c|c|c|c|c|c|}
\hline \multicolumn{3}{|c|}{} & \multicolumn{3}{c|}{ ÍNDICES DESCRIPTIVOS } & \multicolumn{3}{c|}{ ÍNDICES PRÁCTICOS } \\
\hline$X^{2}$ & $p$ & $d f$ & $X^{2} / d f$ & $N N F I$ & CFI & RMSEA & NFI & IFI & MFI \\
\hline 55.600 & 0.156 & 46 & 1.210 & 0.960 & 0.978 & 0.042 & 0.903 & 0.982 & 0.980 \\
\hline
\end{tabular}




\section{Discusión y Conclusiones}

Oportunamente habíamos mencionado que la relevancia de las relaciones entre enseñanza, aprendizaje, uso de tecnologías y rendimiento académico viene dada por su impacto en el desarrollo humano de los países, su importancia en la agenda pública, la invisibilidad del tema, su vacancia científica, la trascendencia local y el interés propio. Este estudio ha corroborado el potencial que tienen las actividades formativas realizadas con TIC, puesto que permiten: estructurar, compartir y difundir el conocimiento -superando las barreras de tiempo y espacio-, basado en la personalización y el acompañamiento del estudiante, ya que el aprendizaje es un hecho que ocurre en una persona, tiempo y espacio determinado.

La determinación de transformar el aula docente tradicional en un espacio de actividades formativas en el que se utilizan materiales digitales ha dado lugar a desarrollar investigación científica que, en nuestro ámbito, permitió contribuir básicamente en aspectos de la disciplina contable relacionados con: a) estudios en el área pedagógica (implementación de enseñanza en MSP y recursos didácticos multimedia), y b) respuestas al problema del rendimiento académico (el modelo estadístico propuesto permitió medir la satisfacción en virtud de la implementación de nuevas estrategias de enseñanza mediadas por TIC y su resultado en el mejoramiento del desempeño cognitivo).

Según los datos obtenidos del sistema oficial SIU Guaraní, el $79.70 \%$ de la muestra ha logrado calificación teórica de aprobación; esto significa que si se empleara la escala institucional de evaluaciones, la mayoría de los participantes de este estudio ha alcanzado notas que van desde Aprobado (6) hasta Sobresaliente (10); mientras que el rendimiento académico modal se ubicó en siete (7) puntos. Estos resultados dejan en evidencia que, en gran número, los estudiantes de la MSP han obtenido calificaciones que les permite promover la asignatura.

De manera similar, estos resultados apoyan las conclusiones a las que llegan García-Martín y Cantón-Mayo (2019), quienes muestran con sus hallazgos que existe una relación entre el uso de las tecnologías y el rendimiento académico. El estudio de estas autoras apuntó a la indagación del impacto de varias herramientas tecnológicas en estudiantes adolescentes en cuatro asignaturas y sus datos evidencian un aumento del rendimiento en Ciencias y en áreas de lingüística con herramientas relacionadas con los motores de búsqueda, y en Matemáticas por el uso de podcast.

La medición del grado de satisfacción de los estudiantes ante la modalidad semipresencial y el impacto en su rendimiento académico abordado mediante estructuras de covarianza fue una decisión correcta, puesto que ha posibilitado generar el planteo de un modelo estadístico -a partir de variables psicoeducativas- que podría ser un recurso válido para medir con eficacia propuestas de intervención educativa universitaria en ámbitos tecnológicos. En efecto, la eficacia de este modelo consiste en mostrar la relación (dirección, sentido y magnitud) entre los factores predictivos enseñanza, materiales y aprendizaje, mediados por TIC, y el rendimiento académico de estudiantes universitarios, en el ámbito de la asignatura CB.

Las estimaciones de las cargas factoriales, como de los parámetros estructurales del modelo, también de los coeficientes que evalúan la covarianza entre los errores $\mathrm{E}$, resultaron en todos los casos valores estadísticamente significativos (algunos para $p<0.05$, y otros para $p<0.01$ ). Por lo tanto, desde el punto de vista analítico, podemos sostener que las medidas estimadas fueron correctas; no obstante, sería justo reconocer que los resultados conseguidos presentan ciertas limitaciones. Evidentemente, hace falta insistir en futuras 
investigaciones en el aporte de nuevas variables, o las analizadas en este trabajo pero medidas de manera diferente, que hagan posible mejorar el porcentaje de varianza explicada en la variable respuesta.

Entre los aportes que entendemos realiza este trabajo se encuentra el hecho de que en el modelo contrastado el factor explicativo materiales se halla conformado por variables dinámicas (material de lectura, cuestionarios, tareas y foros) que son susceptibles de intervención. Así, de acuerdo con lo señalado en el párrafo anterior, en caso de observarse necesario podrían ser modificadas a efectos que resulten de mayor utilidad para explicar la variabilidad del rendimiento académico ( $p$. ej., incorporar o quitar actividades en el aula virtual).

Otra cuestión que deseamos destacar de la modelización estadística propuesta (véase Figura 2), es que la variable latente independiente enseñanza es la que mayor protagonismo posee a la hora de explicar el rendimiento en Contabilidad; puesto que no solo incide en forma directa, sino que lo hace de manera indirecta a través de los factores mediadores materiales $y$ aprendizaje. En efecto, estos factores influyen en la variable dependiente, el primero de los dos últimos (materiales) a través de aprendizaje, mientras que ambos (materiales y aprendizaje) lo hacen también en forma directa.

Una de las dificultades habituales en la elaboración de modelos teóricos reside en que estos deben ser sencillos pero a la vez reflejar la realidad que suele ser compleja. Es sabido que cuando en un modelo interviene un número elevado de variables se obtiene una representación complicada; sin embargo, la opción de prescindir de algunas de ellas puede suponer que se ha dejado de tener en cuenta aspectos importantes del fenómeno objeto de análisis. Evidentemente, definir la simplicidad o parsimonia de los modelos es una tarea dificultosa, pues lo que se gana en sencillez, puede perderse en eficiencia al representar el escenario que se analiza. En los modelos estadísticos el número de parámetros que se estiman es una buena medida de la complejidad del modelo. (Closas, 2011).

El modelo planteado puede ser mejorado, pero se considera que es un paso adelante en el estudio de esta problemática, que se anhela pueda servir como referencia para futuras investigaciones que se realicen en esta línea. Ahora bien, dado que los participantes fueron alumnos pertenecientes a un centro académico específico, así como a una carrera, asignatura y modalidad de enseñanza determinada, algunas evidencias alcanzadas en este trabajo ( $p$. ej., el porcentaje de jóvenes que ha logrado calificación teórica de aprobación) no sería conveniente extenderse a otros estudiantes universitarios, menos aún hacer inferencias sobre poblaciones no representadas en la muestra, a pesar que la misma fue seleccionada de manera aleatoria.

En el marco de la temática abordada, entre las actividades que podrían proponerse a efectos de contribuir en el mejoramiento del desempeño académico, se encuentran: a) fomentar la presentación de propuestas educativas que se lleven a cabo en MSP, puesto que los entornos virtuales de aprendizaje tienen características valoradas por los estudiantes (p. ej., hipermedia y ubicuidad); y b) ofrecer capacitación en el manejo de la plataforma Moodle para aquellos jóvenes que realizan una primera experiencia en comunidades de aprendizaje en línea.

Estas recomendaciones van acordes con el estudio de Zempoalteca et al. (2017), cuya investigación midió el impacto en el rendimiento de universitarios que conocen los ambientes virtuales y participan en ellos motivados por docentes que fomentan el uso adecuado de las tecnologías. Los autores llegan a la conclusión de que el uso innovador de las TIC tiene efecto favorable en la práctica académica de los estudiantes, 
siempre y cuando los docentes hagan un uso significativo de estos recursos.

Más allá de los aciertos e inconvenientes que conlleva el desarrollo de toda investigación, aspiramos a que este primer acercamiento al análisis del fenómeno educativo-tecnológico haya evidenciado que la incorporación de TIC no limita ni menoscaba la actuación del docente, pues descansa en él la responsabilidad de determinar y apreciar las condiciones particulares de la intervención didáctica al decidir cuál es la mejor combinación posible (contenidos, actividades y materiales) para lograr los objetivos educativos que persigue en su asignatura. En sintonía con lo anterior concluyen García-Martín y Cantón-Mayo (2019), quienes recomiendan que los docentes deben seleccionar cuidadosamente las herramientas tecnológicas y las actividades en función de los contenidos, de esta manera, los estudiantes utilizarán las tecnologías con eficacia y estas ejercerán influencias positivas para su rendimiento.

En el contexto sociocultural de origen de la muestra, este estudio ha aportado algo más de claridad sobre la relación entre la satisfacción de los estudiantes con la modalidad semipresencial, las estrategias implementadas y el rendimiento con el fin de posibilitar la apertura de diálogos sobre medidas de intervención psicopedagógicas y la adopción de decisiones institucionales que permitan promover soluciones válidas, al menos, a las dificultades habituales que actualmente se presentan en el rendimiento de la asignatura CB.

Troncoso et al. (2010) han demostrado la eficacia de la modalidad semipresencial implementada en un curso de Matemáticas, ya que el b-learning permitió combinar tecnología, pedagogía y una comunicación permanente, lo cual dio como resultado un mayor rendimiento de los estudiantes. Además, posibilitó promover el desarrollo de competencias genéricas como el pensamiento crítico y las destrezas de comunicación por el uso permanente de los foros y la interacción con los docentes y compañeros, actividades "valiosas para el futuro profesional del estudiante en un mundo actual, pues se fomenta la colaboración en los ambientes virtuales facilitando cambios de actitudes sociales" (p. 25).

Como reflexión final, consideramos que la incorporación de aplicaciones web al proceso educativo requiere de docentestutores con nuevas habilidades, no siempre comparables con las utilizadas en los sistemas presenciales. Las innumerables posibilidades de contacto con el conocimiento a través de medios tecnológicos no garantiza el acceso a los saberes; son las actividades que los docentes Ilevan a cabo con TIC lo que brinda el incremento de los resultados de aprendizaje y el mejoramiento del rendimiento académico. Esta consideración se encuentra en total armonía con lo que expresa el modelo estadístico propuesto, en el sentido que puede observarse que los constructos enseñanza y materiales son los que mayor relevancia operativa y funcional tienen como determinantes del desarrollo académico de los estudiantes en prácticas educativas semipresenciales.

\section{Referencias}

Bentler, P. M. (2006). EQS Structural equations program manual. Encino, CA: Multivariate Software, Inc.

Bentler, P. M. y Weeks, D. G. (1980). Linear structural equations with latent variables. Psychometrika, 45, 289-308.

Bentler, P. M. y Wu, E. J. (2015). Supplement to EQS 6.3 for Windows User's Guide. Encino, CA: Multivariate Software, Inc.

Closas, A. H. (2011). Ecuaciones estructurales, una técnica estadística para formular y contrastar modelos de relaciones causa-efecto. Actualidad y Prospectiva, Revista de la Facultad de Ciencias Económica - UNNE, 7(1), 175-189. 
Chávez, F. (2018). Estrategias de aprendizaje y rendimiento académico de los estudiantes del III ciclo de Contabilidad de la Universidad Católica Los Ángeles de Chimbote, Ayacucho (Tesis de maestría). Universidad Católica Los Ángeles de Chimbote, Perú.

De Castro, I. G., Closas, A. H. y Cruz, R. T. (2019). Modelo didáctico para la intervención educativa universitaria, en contabilidad y en ambientes tecnológicos. Revista de la Facultad de Ciencias Económica - UNNE, 22(1), 65-90. http://dx.doi.org/10.30972/rfce. 2213949

De la Fuente, J. y Martínez, J. M. (2004). Escalas para la Evaluación Interactiva del Proceso de Enseñanza-Aprendizaje, EIPEA. Madrid: EOS.

Fox, D. (1981). El proceso de investigación en educación. Pamplona, España: EUNSA

Gallardo, E. E., Marqués, L. y Bullen, M. (2014). Usos académicos y sociales de las tecnologías digitales del estudiante universitario de primer año. Tendencias pedagógicas, No. 23.

García, F., Portillo, J. Romo J. y Benito, M. (2007). Nativos digitales y modelos de aprendizaje. Actas del IV Simposio Pluridisciplinar sobre Diseño, Evaluación y Desarrollo de Contenidos Educativos Reutilizables (SPDEDE). Bilbao. Universidad del País Vasco.

García-Martín, S. y Cantón-Mayo, I. (2019). Uso de tecnologías y rendimiento académico en estudiantes adolescentes.
Comunicar, 27(59), 73-81. https://doi.org/ 10.3916/C59-2019-07

Nicolás, J. L. (2018). Conocimientos previos de Contabilidad Básica y el desarrollo de capacidades para el registro de prácticas contables en estudiantes universitarios (Tesis de maestría). Universidad San Martín de Porres, Lima, Perú.

Satorra, A. y Bentler, P. M. (1988). Scaling corrections for statistics in covariance structure analysis. Los Angeles, CA: UCLA Statistics Series 2.

Schermelleh-Engel, K., Moosbrugger, H. y Müller, H. (2003). Evaluating the Fit of Structural Equation Models: Tests of Significance and Descriptive Goodnessof-Fit Measures. Methods of Psychological Research Online, 8(2), 23-74.

Troncoso, O., Cuicas, M. y Debel, E. (2010). El modelo b-learning aplicado a la enseñanza del curso de Matemática I en la carrera de Ingeniería Civil. Actualidades Investigativas en Educación, 10(3), 1-28. https://www. redalyc.org/pdf/447/44717980015.pdf

Zabalza, M. A. (2002). La enseñanza universitaria. El escenario y sus protagonistas. Madrid: Narcea.

Zempoalteca, B., Barragán, J. F., González, J. y Guzmán, T. (2017). Formación en TIC y competencia digital en la docencia en instituciones públicas de educación superior. Apertura, 9(1). http://dx.doi.org /10.18381/Ap.v9n1.922 